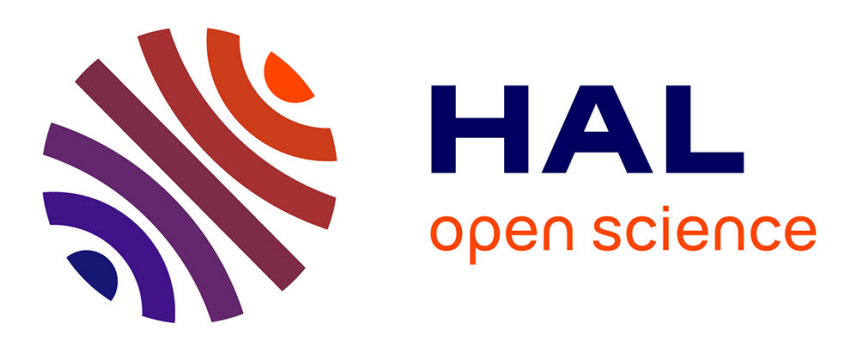

\title{
Les médicaments cardiovasculaires qui favorisent le diabète
}

\author{
B. Vergès
}

\section{To cite this version:}

B. Vergès. Les médicaments cardiovasculaires qui favorisent le diabète. Archives des Maladies du Coeur et des Vaisseaux - Pratique, 2019, 2019, pp.20 - 23. 10.1016/j.amcp.2018.11.004 . hal03486212

\section{HAL Id: hal-03486212 \\ https://hal.science/hal-03486212}

Submitted on 20 Dec 2021

HAL is a multi-disciplinary open access archive for the deposit and dissemination of scientific research documents, whether they are published or not. The documents may come from teaching and research institutions in France or abroad, or from public or private research centers.
L'archive ouverte pluridisciplinaire HAL, est destinée au dépôt et à la diffusion de documents scientifiques de niveau recherche, publiés ou non, émanant des établissements d'enseignement et de recherche français ou étrangers, des laboratoires publics ou privés.

\section{다)(1) $(5$}

Distributed under a Creative Commons Attribution - NonCommercial| 4.0 International 


\section{DOSSIER / FICHE TECHNIQUE}

\section{Les médicaments cardiovasculaires qui favorisent le diabète}

\section{Cardiovascular drugs promoting diabetes}

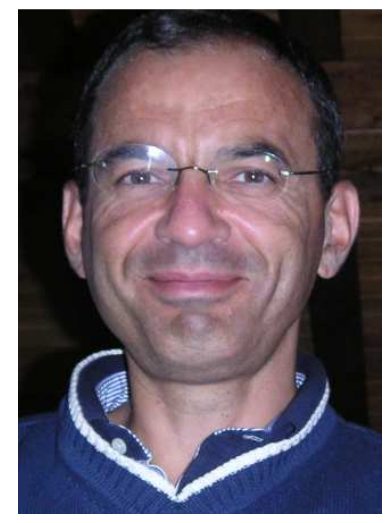

\section{$B$. Vergès ${ }^{a, b}$}

a. Service Endocrinologie, Diabétologie et Maladies Métaboliques, Hôpital du Bocage, centre hospitalier universitaire de Dijon, 2 boulevard du Maréchal de Lattre de Tassigny, 21079 Dijon cedex, France.

b. INSERM LNC-UMR 1231, Université de Bourgogne, AgroSup, UFR des Sciences de Santé, 7 bd Jeanne d'Arc, 21000 Dijon, France.

bruno.verges@chu-dijon.fr

Le diabète de type 2 est une maladie extrêmement fréquente caractérisée par un risque élevé de complications microvasculaires sévères et de neuropathie ainsi que par un risque cardiovasculaire très augmenté. II représente donc un problème majeur de santé publique. Parmi les agents thérapeutiques utilisés dans la sphère cardiovasculaire, certains présentent un risque intrinsèque de diabète. II s'agit des thiazidiques des bétabloquants et des statines. Nous analyserons, dans cet article, le risque diabétogène pour chaque classe thérapeutique en le contrebalançant par le bénéfice cardiovasculaire prouvé.

\section{Les thiazidiques}

Les thiazidiques ont un risque diabétogène propre. Leur effet hyperglycémiant est clairement authentifié. Dans une large méta-analyse de 26 études randomisées regroupant 16162 patients hypertendus il est montré que les traitements par thiazidiques augmentent significativement la glycémie à jeun de $0,26 \mathrm{mmol} / \mathrm{l}$, en moyenne [1]. Cet effet apparaît dosedépendant puisque les doses d'hydrochlorothiazide ou de chlortalidone inférieures ou égales à $25 \mathrm{mg} /$ jour augmentent, en moyenne, la glycémie de $0,15 \mathrm{mmol} / \mathrm{l}$ alors que les doses plus élevées entraînent une augmentation de $0,60 \mathrm{mmol} / \mathrm{l}$ [1]. Dans l'étude ALLHAT (Antihypertensive and Lipid-Lowering treatment to prevent Heart Attack Trial), le traitement par le thiazidique, chlortalidone, a entraîné une augmentation moyenne de 0,62 $\mathrm{mmol} / \mathrm{l}$ de la glycémie à jeun, chez les sujets non diabétiques à l'entrée dans l'étude [2]. 
L'augmentation du risque de survenue de diabète sous thiazidiques est mise en évidence dans plusieurs études. Dans l'étude ALLHAT, ayant suivi, pendant plus de 4 ans, 33357 patients hypertendus, le risque de survenue de diabète sous thiazidique (chlortalidone) est de $11,6 \%$, significativement plus élevée que dans le groupe recevant l'amlodipine $(9,8 \%$, $p=0,04)$ ou dans celui recevant du lisinopril $(8,1 \%, p<0,001)$ [2]. Dans l'étude SHEP (Systolic Hypertension in the Elderly Program), au cours de laquelle 4536 patients hypertendus ont reçu de façon randomisée un traitement hypotenseur contenant un thiazidique (chlortalidone) ou un traitement placebo, pendant trois ans, il est observé une augmentation non significative de $20 \%$ du risque de diabète sous thiazidiques [3]. Cependant, lors du suivi prolongé des patients de l'étude SHEP pendant 14,3 années, il est noté une augmentation significative de $56 \%$ de risque de survenue de diabète [4]. Au cours de l'étude NAVIGATOR, ayant suivi pendant cinq ans des patients traités par agents hypotenseurs dont 6346 par diurétiques (très majoritairement thiazidiques), il est observé une augmentation significative du diabète de $23 \%$ [5]. Le risque de diabète sous thiazidiques est dose-dépendante et ainsi, nettement moins marqué avec de faibles doses. Dans l'étude DIME, au cours de laquelle 1130 patients hypertendus japonais ont été randomisés en un traitement par $12,5 \mathrm{mg}$ d'hydrochlorothiazide ou en un traitement hypotenseur non diurétique, il n'est pas objectivé d'augmentation du risque de survenue de diabète durant les 4,4 années de suivi $(4,6 \%$ dans le groupe hydrochlorothiazide versus $4,9 \%$ dans le groupe traitement hypotenseur non diabétique) [6]. II est vraisemblable que l'effet hyperglycémiant des thiazidiques soit différent selon les molécules. En effet, les données de plusieurs études et méta-analyses indiquent que l'effet diabétogène est plus marqué pour l'hydrochlorothiazide que pour la chlortalidone ou l'indapamide [7].

Plusieurs mécanismes semblent expliquer l'effet hyperglycémiant des thiazidiques. II a été montré, au moyen de clamps euglycémiques hyperinsuliniques, une diminution significative de la sensibilité à l'insuline sous hydrochlorothiazide [8]. Cet effet pourrait être secondaire à l'augmentation des catécholamines induite par les thiazidiques. En outre, il a été montré que les thiazidiques réduisaient la sécrétion d'insuline chez les sujets sains [9]. Cet effet pourrait passer par l'hypokaliémie induite par les thiazidiques, qui diminue la réponse insulinique pancréatique au glucose. II a, en effet, été retrouvé, dans une large méta-analyse de 59 études cliniques, une corrélation négative franche entre la kaliémie et la glycémie sous traitement thiazidique [10]. En outre, il a été montré que la prévention de l'hypokaliémie par apport de potassium per os, chez les patients sous thiazidiques, réduisait significativement l'effet diabétogène de cette classe thérapeutique [11].

La question de savoir si l'accroissement du risque de diabète sous thiazidiques augmente le risque cardiovasculaire est controversée. Dans l'étude ALLHAT, malgré une incidence plus élevée de diabète dans le groupe thiazidique comparé à l'amlodipine et au lisinopril, le risque d'événements cardiovasculaires n'était pas différent entre les trois types de traitement [2]. II a même été montré, avec quatre années de suivi supplémentaire, que les patients ayant développé un diabète sous thiazidiques présentaient un risque plus faible de survenue d'événements cardiovasculaires que ceux ayant présenté un diabète sous amlodipine ou lisinopril [12]. Dans l'étude SHEP, il était observé que les diabètes apparus sous placebo étaient associés une augmentation du risque cardiovasculaire alors que ceux développés sous thiazidiques ne l'étaient pas [3]. En revanche, plusieurs études, avec un suivi prolongé de 18 à 25 ans, ont montré que les diabètes induits par les traitements hypotenseurs étaient associés à une augmentation significative du risque cardiovasculaire [12]. Cependant, il ne faut pas oublier que, chez les patients diabétiques, les thiazidiques ont fait la preuve de leur efficacité pour réduire significativement le risque d'événements cardiovasculaires comparés au placebo et qu'ils permettent une diminution du risque cardiovasculaire comparable à celle observée avec les autres agents hypotenseurs [7]. 
Si les thiazidiques ont un effet diabétogène incontestable, leur bénéfice cardiovasculaire dépasse largement leur effet délétère sur la glycémie. Leur effet hyperglycémiant est limité à faible dose. Ainsi, cette classe thérapeutique continue à jouer un rôle important dans le traitement hypotenseur, chez les patients diabétiques.

\section{Bétabloquants}

Il est observé une augmentation du risque de diabète sous traitement par bétabloquants comme l'attestent de nombreuses études cliniques. Ainsi, dans une étude prospective réalisée chez 12550 adultes non diabétiques, âgés de 45 à 64 ans, suivis pendant 3 à 6 ans, il est observé une augmentation du risque de diabète de $28 \%$ comparé aux autres traitements hypotenseurs après ajustement pour l'âge, le sexe, l'ethnie, le niveau d'éducation, l'adiposité, les antécédents familiaux de diabète et le niveau d'activité physique (RR = 1, 28 [1,04 - 1,57]) [13]. Dans l'étude LIFE (Losartan Intervention For End-point reduction), au cours de laquelle 9193 patients hypertendus âgés de 55 à 80 ans ont été traités, après randomisation, soit par aténolol soit par losartan, il était observé une augmentation significative de $25 \%$ du risque de survenue de diabète chez les sujets sous aténolol durant un suivi moyen de 4,8 années [14]. Dans l'étude NAVIGATOR, il était noté une augmentation de $10 \%$ du risque de diabète sous bétabloquants qui, cependant, n'atteignait pas le seuil de significativité statistique [5]. Le risque diabétogène des bétabloquants apparaît clairement dans la méta-analyse de Bangalore et al, réalisée à partir du 12 études contrôlées regroupant 94492 patients hypertendus, dans laquelle le traitement par bétabloquants comparé au placebo était associé à une augmentation significative de survenue de diabète de $33 \%$ ( $R R=1,33[1,00-1,76])$ [15]. Dans cette méta-analyse, le risque apparaît plus prononcé chez les patients âgés de plus de 60 ans ( $R R=2,13[1,34$ $3,38]$ ). Le traitement par bétabloquants est associé à une augmentation significative du risque de diabète de $23 \%$ en comparaison avec les inhibiteurs d'enzyme de conversion, de $19 \%$ en comparaison avec les antagonistes des récepteurs de l'angiotensine-Il et de $21 \%$ en comparaison avec les inhibiteurs calciques [15]. En revanche, lorsque les bétabloquants sont comparés aux thiazidiques, le risque de survenue de diabète est significativement réduit de $26 \%$, ce qui apparaît indiquer un effet diabétogène supérieur des thiazidiques comparés aux bétabloquants [15]. II faut noter que le traitement combiné par bétabloquants et thiazidiques augmente très significativement le risque de diabète comme l'indique une large méta-analyse publiée en 2005 [16].

Les données de la méta-analyse de Bangalore et al suggèrent un risque diabétogène plus élevé avec l'aténolol, qui apparaît associé à une augmentation significative de diabète, comparé au métoprolol et au propranolol pour lesquels l'augmentation du risque de diabète n'atteint pas la significativité statistique [15]. Cependant, il faut noter que dans cette métaanalyse, le nombre de patients traités par aténolol était significativement plus important que ceux recevant du métoprolol ou du propranolol, ce qui pourrait expliquer ces différences.

Le risque de diabète sous bétabloquant augmente avec la durée du traitement et l'IMC initial est un facteur indépendant de survenue de diabète [15].

Plusieurs mécanismes sont mis en avant pour expliquer l'effet diabétogène des bétabloquants. Dans des études ayant utilisé le clamp euglycémique hyperinsulinique, il a été noté une diminution significative de la sensibilité à l'insuline sous propranolol, sous aténolol et sous métoprolol $[17,18]$. Une diminution de l'oxydation de glucose et de la thermogenèse est aussi objectivée sous bétabloquants [17]. Par ailleurs, une légère diminution de la sécrétion d'insuline est observée sous bétabloquants (18). La diminution de la sensibilité à l'insuline sous bétabloquants pourrait s'expliquer par une réduction du flux sanguin musculaire réduisant ainsi le glucose disponible pour le muscle (et plus particulièrement les fibres musculaires de type I très insulinosensible) et par une diminution de l'effet stimulateur des catécholamines sur la thermogenèse et la consommation d'énergie. 
La prise de poids observée sous bétabloquants pourrait aussi intervenir comme facteur diabétogène [19]. La réduction de sécrétion d'insuline s'explique via le blocage des récepteurs $\beta 2$, qui stimulent la sécrétion d'insuline [20].

\section{Statines}

Au cours des dernières années, une large littérature s'est développée au sujet des diabètes induits par les statines. Au cours de l'étude NAVIGATOR, il est observé une augmentation significative de survenue de diabète de $32 \%$ chez les sujets recevant un traitement par statines $(\mathrm{OR}=1,32[1,14-1,48])$ [5]. L'effet diabétogène des statines a été confirmé par la méta-analyse de Sattar et al réalisée à partir de 13 études contrôlées regroupant 91140 patients, dans laquelle il était objectivé une augmentation de $9 \%$ du risque de survenue de diabète, sous statines, sur une durée moyenne de quatre ans $(O R=1,09[1,02-1,17])$ [21]. II ressort de cette méta-analyse que pour 225 patients traités par statines pendant quatre ans, un cas de diabète sera observé. Le risque de survenue de diabète sous traitement par statines est associé à l'âge, au BMI et à l'importance de la réduction du LDL cholestérol. Ce dernier point suggère un risque de diabète d'autant plus élevé que l'effet hypocholestérolémiant de la statine est important. A ce titre, il faut noter que dans cette méta-analyse, les études ayant utilisé des statines de faible intensité (lovastatine, pravastatine) étaient majoritaires avec une possible sous-estimation du risque de diabète induit par statines. Une méta-analyse plus large, réalisée à partir de 29 études contrôlées, dont 12 avec des statines puissantes (atorvastatine, rosuvastatine), regroupant 163039 patients, objective un risque de $12 \%$ de survenue du diabète sur un suivi moyen de 4,8 années [22]. Dans cette méta-analyse, le risque de diabète était significativement plus élevé avec l'atorvastatine $(+34 \%, \mathrm{OR}=1,34[1,14-1,57])$ et avec la rosuvastatine $(+17 \%, \mathrm{OR}=$ $1,17[1,02-1,35])$ [22]. D'une façon générale, le risque de diabète apparaît significativement plus élevé avec les statines les plus puissantes. Dans l'étude TNT (Treat to New Targets), au cours de laquelle l'atorvastatine $10 \mathrm{mg}$ a été comparé à l'atorvastatine $80 \mathrm{mg}$ chez cette 1595 sujets coronariens stables, il était noté une augmentation non significative de $10 \%$ du risque de diabète sous atorvastatine $80 \mathrm{mg}$ [23]. Dans l'étude IDEAL (Incremental Decrease in End points through Aggressive Lipid lowering), au cours de laquelle l'atorvastatine $80 \mathrm{mg}$ a été comparé à la pravastatine $20 \mathrm{mg}$, en post-infarctus du myocarde, il était observé une augmentation du risque de diabète de $19 \%$ avec l'atorvastatine $80 \mathrm{mg}$, à la limite de la significativité statistique $(p=0,07)$ [23]. Dans l'étude SPARCL (Stroke Prevention by Aggressive Reduction in Cholesterol Levels), au cours de laquelle un traitement par atorvastatine $80 \mathrm{mg}$ a été comparé à un placebo chez 3807 patients suivis pendant 4,9 années, il était observé une augmentation significative de survenue de diabète de $34 \%$ (OR $=1,34[1,05-1,71])$ [23]. Le risque diabète induit en fonction de l'intensité du traitement par statines a été étudié dans une méta-analyse réalisée à partir de cinq études contrôlées chez 32752 patients [24]. II ressort que le traitement intensif par statines est associée à une augmentation de $12 \%$ du risque de diabète comparé au traitement modéré par statines (OR $=1,12[1,04-1,22])[24]$.

Le risque de diabète sous statines apparaît très sensiblement favorisé par le profil des patients. En effet, dans la méta-analyse de Sattar et al, le risque de survenue de diabète est associé à l'âge, au BMI [21]. L'analyse combinée des études SPARCL, TNT et IDEAL fait ressortir quatre facteurs prédictifs de survenue de diabète sous statines : une glycémie basale supérieure à $1,00 \mathrm{~g} / \mathrm{l}$, un IMC supérieur à 30 , la présence d'une hypertension artérielle et des triglycérides supérieures à $1,50 \mathrm{~g} / \mathrm{l}$. Chez les sujets n'ayant aucun de ces quatre facteurs, le risque de survenue de diabète sous statines est inférieur à $2 \%$ alors que chez ceux ayant les quatre facteurs le risque est supérieur à $25 \%$ [23]. Ainsi, le risque de survenue de diabète sous statines est surtout réel chez les patients présentant un syndrome métabolique. 
Cependant, le risque de survenue de diabète sous statines ne doit pas faire oublier le large bénéfice cardiovasculaire entraîné par cette classe thérapeutique. Le bénéfice cardiovasculaire des statines, chez les patients diabétiques, est largement confirmé. Dans l'étude CARDS, réalisée spécifiquement chez des patients diabétiques de type 2 , le traitement par atorvastatine a permis une réduction très significative de $37 \%$ des événements cardiovasculaires majeurs, sur un suivi moyen de 3,9 années [25]. Dans une large méta-analyse, ayant évalué l'effet du traitement par statines chez 18686 patients diabétiques, à partir de 14 études contrôlées, il apparaît que pour chaque réduction du LDL cholestérol de $1 \mathrm{mMol} / \mathrm{l}(0,39 \mathrm{~g} / \mathrm{l})$ il est obtenu une réduction significative de $21 \%$ des événements cardiovasculaires majeurs [26]. Dans le sous-groupe de 1501 patients diabétiques de l'étude TNT, la réduction des événements cardiovasculaires majeurs était significativement plus importante sous atorvastatine $80 \mathrm{mg}$ que sous atorvastatine $10 \mathrm{mg}$ $(\mathrm{HR}=0,75[0,58-0,97], \mathrm{p}=0,026)$ [23]. Enfin, dans la méta-analyse ayant analysé le risque de diabète en fonction de l'intensité du traitement, il apparaît que le risque de survenue d'événements cardiovasculaires majeurs est significativement diminué de $16 \%$ chez les patients ayant reçu un traitement intensif par statines comparé à ceux ayant reçu un traitement d'intensité modérée [24].

Les mécanismes responsables du diabète induit sous statines demeurent mal précisés. La diminution des isoprénoïdes, secondaire à l'inhibition de l'HMGCoA réductase, est suspectée modifier significativement le métabolisme glucidique. II a été montré, in vitro, que la réduction des isoprénoïdes sous atorvastatine réduisait l'expression du transporteur au glucose GLUT4 dans les adipocytes [27]. Par ailleurs, l'inhibition de I'HMGCoA réductase s'accompagne d'une diminution du coenzyme Q10 susceptible de réduire la concentration d'ATP dans la cellule pancréatique et ainsi de diminuer la sécrétion d'insuline [27]. II a été objectivé sous rosuvastatine et simvastatine une diminution plasmatique de l'adiponectine qui est une adipokine augmentant la sensibilité à l'insuline. Ce mécanisme pourrait donc être aussi en cause dans l'effet diabétogène des statines. L'effet musculaire des statines pourrait aussi être impliqué. En effet, il est observé sous statines une dysfonction mitochondriale de la cellule musculaire susceptible d'entraîner une insulinorésistance locale [27]. Par ailleurs, la fatigabilité musculaire, parfois observée sous statines, pourrait favoriser l'insulinorésistance musculaire.

\section{Conclusion}

Les thiazidiques, les bétabloquants et les statines augmentent significativement le risque de diabète. Cependant, leurs bénéfices cardiovasculaires dépassent largement cet inconvénient. D'un point de vue pratique, le risque de survenue de diabète sous thiazidiques peut être réduit par l'utilisation de faibles doses et la correction de l'hypokaliémie. Chez les patients recevant un traitement par bétabloquant ou statines, il apparaît conseillé de surveiller régulièrement leur glycémie et de mettre en place des mesures hygiéno-diététiques préventives chez les patients présentant un syndrome métabolique, afin de réduire le risque de survenue de diabète induit par ces classes thérapeutiques.

\section{Déclaration de liens d'intérêts}

L'auteur déclare ne pas avoir de liens d'intérêts.

\section{Références}

[1] Zhang X, Zhao Q. Association of Thiazide-Type Diuretics With Glycemic Changes in Hypertensive Patients: A Systematic Review and Meta-Analysis of Randomized Controlled Clinical Trials. J Clin Hypertens Greenwich Conn 2016;18:342-51.

[2] ALLHAT Officers and Coordinators for the ALLHAT Collaborative Research Group. The Antihypertensive and Lipid-Lowering Treatment to Prevent Heart Attack Trial. Major outcomes in high-risk hypertensive patients randomized to angiotensin- 
converting enzyme inhibitor or calcium channel blocker vs diuretic: The Antihypertensive and Lipid-Lowering Treatment to Prevent Heart Attack Trial (ALLHAT). JAMA 2002;288:2981-97.

[3] Savage PJ, Pressel SL, Curb JD, et al. Influence of long-term, low-dose, diureticbased, antihypertensive therapy on glucose, lipid, uric acid, and potassium levels in older men and women with isolated systolic hypertension: The Systolic Hypertension in the Elderly Program. SHEP Cooperative Research Group. Arch Intern Med 1998;158:741-51.

[4] Kostis JB, Wilson AC, Freudenberger RS, et al. Long-term effect of diuretic-based therapy on fatal outcomes in subjects with isolated systolic hypertension with and without diabetes. Am J Cardiol 2005;95:29-35.

[5] Shen L, Shah BR, Reyes EM, et al. Role of diuretics, $\beta$ blockers, and statins in increasing the risk of diabetes in patients with impaired glucose tolerance: reanalysis of data from the NAVIGATOR study. BMJ 2013;347:f6745.

[6] Ueda S, Morimoto T, Ando S-I, et al. A randomised controlled trial for the evaluation of risk for type 2 diabetes in hypertensive patients receiving thiazide diuretics: Diuretics In the Management of Essential hypertension (DIME) study. BMJ Open 2014;4:e004576.

[7] Scheen AJ. Type 2 Diabetes and Thiazide Diuretics. Curr Diab Rep 2018;18:6.

[8] Pollare T, Lithell H, Berne C. A comparison of the effects of hydrochlorothiazide and captopril on glucose and lipid metabolism in patients with hypertension. $\mathrm{N}$ Engl $\mathrm{J}$ Med 1989;321:868-73.

[9] Fajans SS, Floyd JC, Knopf RF, Rull J, Guntsche EM, Conn JW. Benzothiadiazine suppression of insulin release from normal and abnormal islet tissue in man. $\mathrm{J}$ Clin Invest 1966;45:481-92.

[10] Zillich AJ, Garg J, Basu S, Bakris GL, Carter BL. Thiazide diuretics, potassium, and the development of diabetes: a quantitative review. Hypertens Dallas Tex 1979. 2006 Aug;48(2):219-24.

[11] Helderman $\mathrm{JH}$, Elahi D, Andersen DK, et al. Prevention of the glucose intolerance of thiazide diuretics by maintenance of body potassium. Diabetes 1983;32:106-11.

[12] Ong KL, Barter PJ, Waters DD. Cardiovascular drugs that increase the risk of newonset diabetes. Am Heart J 2014;167:421-8.

[13] Gress TW, Nieto FJ, Shahar E, Wofford MR, Brancati FL. Hypertension and antihypertensive therapy as risk factors for type 2 diabetes mellitus. Atherosclerosis Risk in Communities Study. N Engl J Med 2000;342:905-12.

[14] Dahlöf B, Devereux RB, Kjeldsen SE, et al. Cardiovascular morbidity and mortality in the Losartan Intervention For Endpoint reduction in hypertension study (LIFE): a randomised trial against atenolol. Lancet Lond Engl 2002;359:995-1003.

[15] Bangalore S, Parkar S, Grossman E, Messerli FH. A meta-analysis of 94,492 patients with hypertension treated with beta blockers to determine the risk of new-onset diabetes mellitus. Am J Cardiol 2007;100:1254-62.

[16] Mason JM, Dickinson HO, Nicolson DJ, Campbell F, Ford GA, Williams B. The diabetogenic potential of thiazide-type diuretic and beta-blocker combinations in patients with hypertension. J Hypertens 2005;23:1777-81.

[17] DeFronzo RA, Thorin D, Felber JP, et al. Effect of beta and alpha adrenergic blockade on glucose-induced thermogenesis in man. J Clin Invest 1984;73:633-9.

[18] Pollare T, Lithell H, Selinus I, Berne C. Sensitivity to insulin during treatment with atenolol and metoprolol: a randomised, double blind study of effects on carbohydrate and lipoprotein metabolism in hypertensive patients. BMJ 1989;298:1152-7.

[19] Rössner S, Taylor CL, Byington RP, Furberg CD. Long term propranolol treatment and changes in body weight after myocardial infarction. BMJ 1990;300:902-3.

[20] Fonseca VA. Effects of beta-blockers on glucose and lipid metabolism. Curr Med Res Opin 2010;26:615-29. 
[21] Sattar N, Preiss D, Murray HM, et al. Statins and risk of incident diabetes: a collaborative meta-analysis of randomised statin trials. Lancet Lond Engl 2010;375:735-42.

[22] Thakker D, Nair S, Pagada A, Jamdade V, Malik A. Statin use and the risk of developing diabetes: a network meta-analysis. Pharmacoepidemiol Drug Saf 2016;25:1131-49.

[23] Waters DD, Ho JE, DeMicco DA, et al. Predictors of new-onset diabetes in patients treated with atorvastatin: results from 3 large randomized clinical trials. J Am Coll Cardiol 2011;57:1535-45.

[24] Preiss D, Seshasai SRK, Welsh P, et al. Risk of incident diabetes with intensive-dose compared with moderate-dose statin therapy: a meta-analysis. JAMA 2011;305:255664.

[25] Colhoun HM, Betteridge DJ, Durrington PN, et al. Primary prevention of cardiovascular disease with atorvastatin in type 2 diabetes in the Collaborative Atorvastatin Diabetes Study (CARDS): multicentre randomised placebo-controlled trial. Lancet Lond Engl 2004;364:685-96.

[26] Cholesterol Treatment Trialists' (CTT) Collaborators, Kearney PM, Blackwell L, et al. Efficacy of cholesterol-lowering therapy in 18,686 people with diabetes in 14 randomised trials of statins: a meta-analysis. Lancet Lond Engl 2008;371:117-25.

[27] Aiman U, Najmi A, Khan RA. Statin induced diabetes and its clinical implications. J Pharmacol Pharmacother 2014;5:181-5. 